\title{
On the aggravating impact and mitigation of heavy non-liner loads on islanded microgrids
}

\author{
Ahmed Mohamed $^{\mathrm{a}^{*}}$, Vahid Salehi $^{\mathrm{b}}$ \\ ${ }^{a}$ City College of the City University of New York, 160 Convent Avenue, New York 10031, USA \\ ${ }^{b}$ Power Analytics Corporation, 10805 Rancho Bernardo Road, Suite 270, San Diego, CA 92127, USA.
}

\begin{abstract}
In this paper, the impacts of heavy loads, with intermittent non-linear nature, on the stability/security of microgrids operating in an islanded mode will be investigated. The microgrid architecture under study is assumed to have a renewable energy source, a storage system and multiple emergency generators. The impact of non-liner loads will be studied during normal operating conditions, and in the case of various contingencies. Moreover, a solution to mitigate the effect of these non-linear loads based on storage distribution will be discussed.
\end{abstract}

Keywords: Islanded-mode, microgrid, pulsed loads, security, stability, storage distribution

\section{Introduction}

The conventional central architecture and control of power systems previously led to cascaded failures, which in turn led to major disruptions and blackouts. Therefore, the transition towards more distributed electric power system architectures, namely microgrids, and distributed power system controls instead of the conventional central technique, became a must [1]. Microgrids are local energy networks that have the capability to operate independently from the grid in an islanded mode whenever needed. In this mode, the microgrid's local controller utilizes local generation and storage to supply higher-priority loads for as much time as possible. Due to the absence of the grid, which normally buffers microgrid disturbances in the grid-connected mode, some particular load dynamics may lead to instability problems in the islanded mode. For instance, these loads may include big motors during starting or a group of plug-in electric vehicles charging at the same time.

Therefore, security assessment and analysis are essential for islanded microgrids. A shipboard power system resembles the concept of a microgrid operating in a smart grid environment where the system is capable of self-diagnosing, self-healing, and self-reconfiguring [2], [3]. Due to the analogy between islanded microgrids and shipboard power systems, and the fact that shipboard power systems are quite subject to heavy non-linear loads, the dynamic security was studied on a shipboard integrated power (IPS) system, as a hybrid AC/DC microgrid example. In these systems, some particular loads draw very high short time current in an intermittent fashion, such as electromagnetic rail weapon, launch systems and free electron lasers. Henceforth, heavy non-linear loads will be referred to collectively as pulsed loads [4].

Shipboard IPSs have the same challenging unique features as islanded microgrids from a power system operation perspective [5]. First of all, IPS is an isolated power system, not supported with a relatively larger grid. Therefore, the system encounters faster dynamics due to the finite generation inertia and is more susceptible to disturbances. Secondly, transients on the load side may cause considerable transients in the system since load/generation ratio is close to 1 . Moreover, the system is physically small

\footnotetext{
* Manuscript received May 14, 2014; revised September 10, 2014.

Corresponding author: Ahmed Mohamed; Tel.: +1 212 650-6619; E-mail address: amohamed@ccny.cuny.edu.

doi: $10.12720 /$ sgce.4.1.30-37
} 
hence the connecting cables have negligible impedance, which may cause instability issues among the different components of the system. Finally, the IPS is more subjected to severe damages, especially during battle conditions, which has to be taken into account while operating security analysis. This is also analogous to the case of an islanded microgrid operating during a natural disaster, e.g. a hurricane.

With the existence of pulsed loads on such systems, dynamic security analysis has to be performed during different contingency conditions and corresponding to different storage distribution techniques. The main security constraints considered are the voltage limits on the AC and DC buses in steady state, and transient conditions during pulsed load occurrence. In addition, the AC voltage frequency is allowed to oscillate only within acceptable limits. Finally, none of the system components, such as cables and transformers, should be overloaded. Table 1 shows the security constraints considered in this study.

Table 1. Security constraints

\begin{tabular}{ll}
\hline \hline Parameter & Secure range \\
\hline AC voltage amplitude & $0.9<\left|V_{a c}\right|<1.1$ \\
AC voltage frequency & $59.5<f<60.5$ \\
Loading of system components & Loading $<100 \%$ \\
DC voltage level & $0.9<V_{d c}<1.1$ \\
\hline \hline
\end{tabular}

\section{Test System Description}

The example system resembles a shipboard power system with scaled down ratings. The system is a hybrid AC/DC system, with zonal distribution architecture on the DC side, as shown in Fig. 1. It includes two $13.8 \mathrm{~kW}$ main generators (MTG) and two $10.4 \mathrm{~kW}$ auxiliary generators (ATG) connected in a ring bus configuration. The bulk of the load consists of two $50 \mathrm{~kW}$ propulsion motors, modeled as permanent magnet machines supplied by PWM drives, with hydrodynamic propeller models as the mechanical load. Each rectifier supplies one of two $0.318 \mathrm{kV}$ DC busses. Furthermore, a photovoltaic (PV) generation system of $10 \mathrm{~kW}$ rated capacity, lithium-ion batteries with 3000 Ah rated capacity and super capacitors (SC) with $200 \mathrm{~F}$ are integrated to the DC microgrid. A PWM controlled DC-DC converter is used as an interface between the PV system and the DC bus. Moreover, a vector decoupling PWM controlled ACDC/DC-AC bidirectional converter was used for connectivity between the AC and DC sides. In the steady state case, the system voltages and loadings are within the normal limits. For transient simulations, we considered a pulse train of four pulses with a rate of $0.2 \mathrm{~Hz}$, a duty ratio of $10 \%$ and amplitude of $20 \mathrm{~kW}$. Each of the four DC zones may include, a pulse load, battery, SC, a normal load or a combination of these elements based on the event studied.

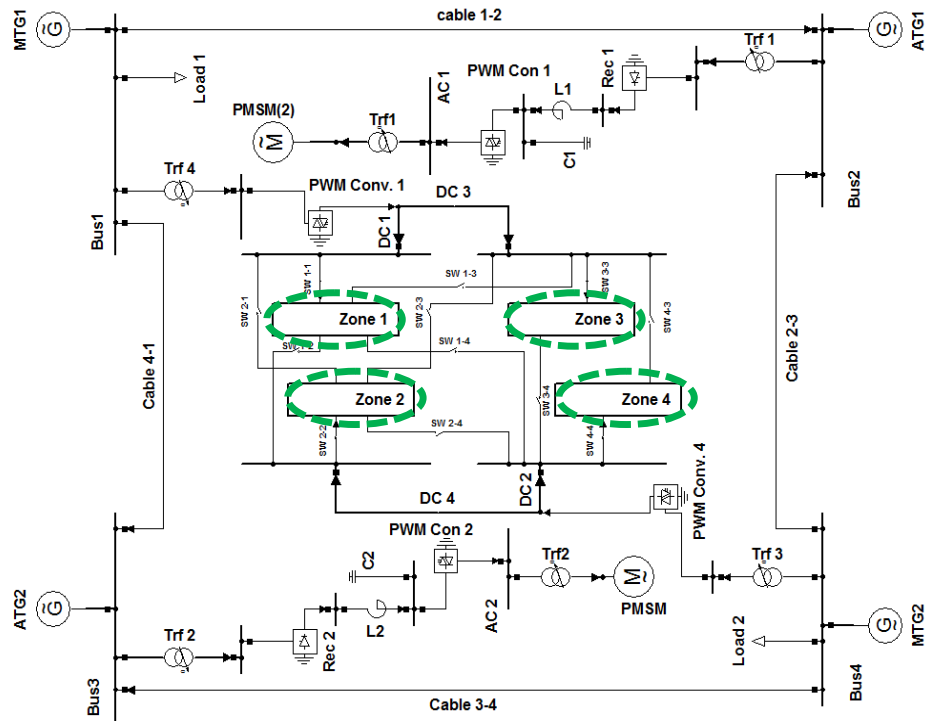

Fig. 1. A single line diagram of the hybrid AC/DC power system under study. 


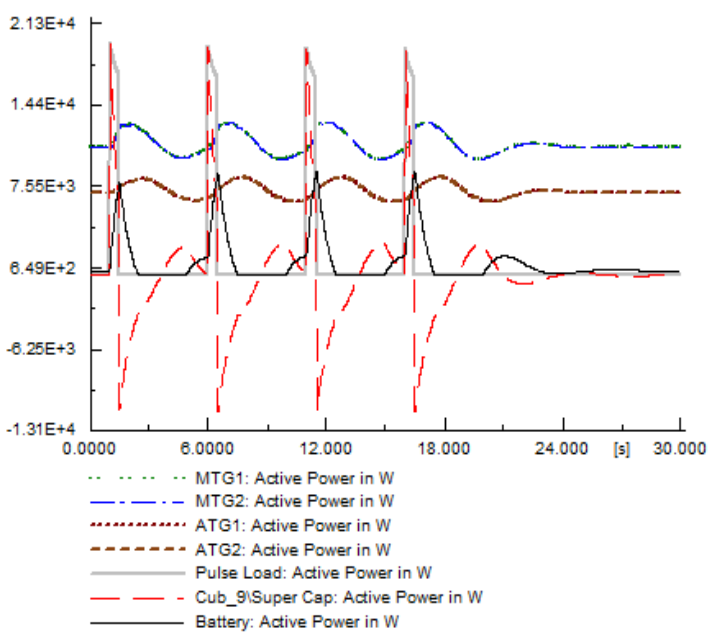

Fig. 2. Output power from each source during pulsed load.
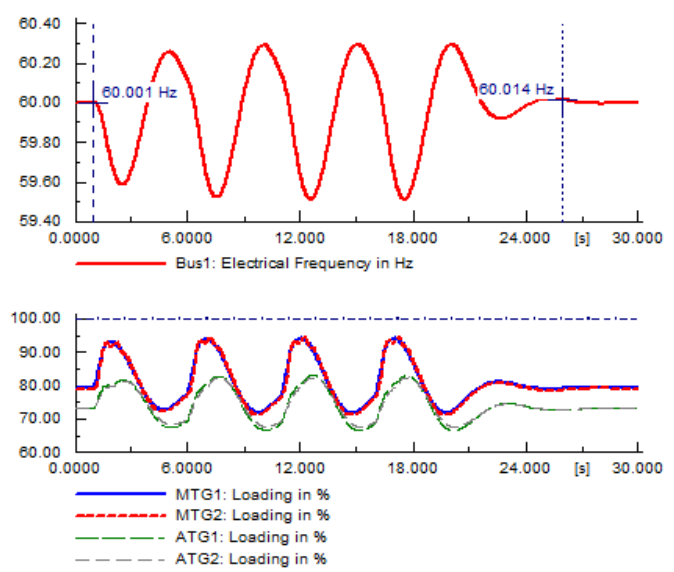

Fig. 4. System frequency and generator loading.
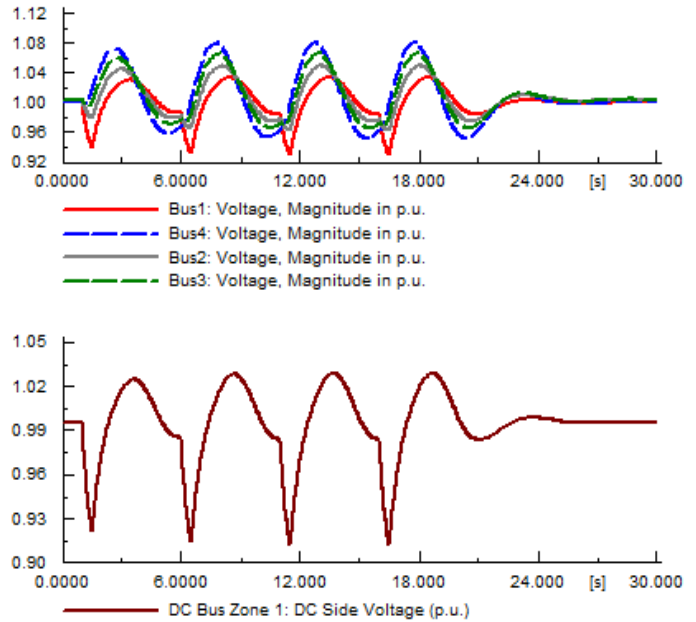

Fig. 3. AC and DC voltages.
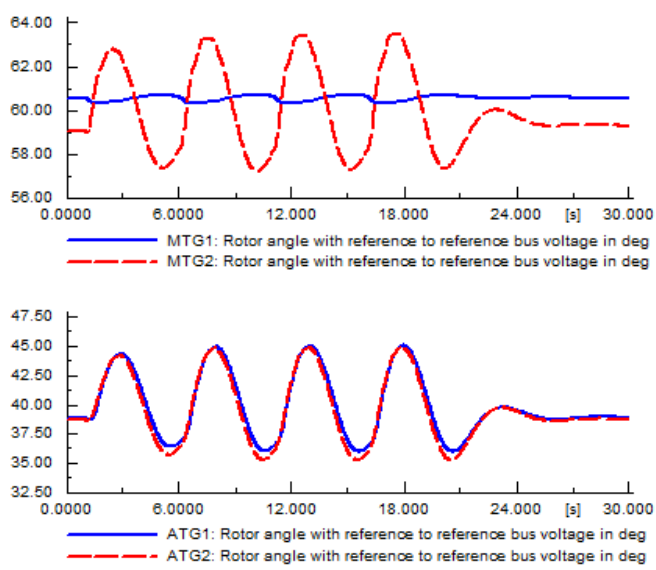

Fig. 5. Rotor angle corresponding to the pulsed load.

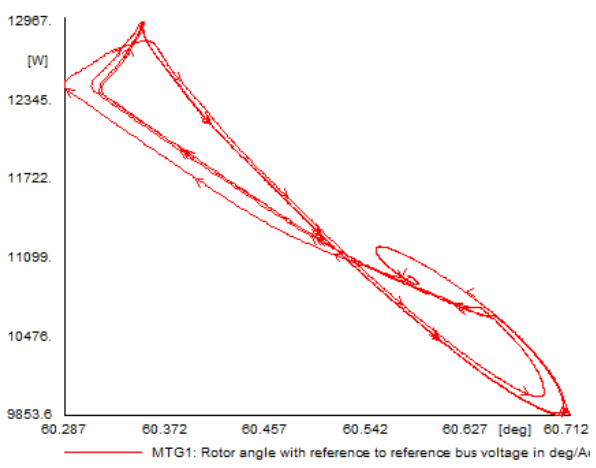

Fig. 6. $P-\delta$ curve.

As shown in Fig. 2, with no contingencies, the super capacitors quickly respond at the beginning of the pulsed load due to its power density, however its power drops and the battery takes over due to its higher energy density. The main and auxiliary generators encounter some swinging and oscillation. Super capacitor and battery are capable of riding the system through the disturbance caused by the pulsed load. The system frequency oscillations are within the $\pm 0.5 \mathrm{~Hz}$ limit, and we have no overloading at any generator according to Fig. 3. Transient values of the voltage are within the limits according to Fig. 4, and 
in addition, the steady state values of voltages are within the limits. Generators' rotor angles and MTG1's power delta-curve are shown in Figs. 5 and 6.

\section{Dynamic Security Analysis}

The security analysis that was performed aimed at studying the effects of:

- System contingencies: in which the system security will be examined under several outage conditions along with the pulsed load.

- Storage distribution: in which the effect of distributing the battery storage system (for thermal management, increased reliability, etc) was examined during the occurrence of pulse loads.

A list of the studied events is shown in Table 2. The first set of events is related to contingencies of the main elements during the pulsed load occurrence. The pulsed load consists of 4 pulses, while the contingency of the equipment takes place after the second pulse, i.e. after $9 \mathrm{~s}$. The pulsed load is placed at zone 1 , and this zone includes the super capacitor and battery storage. The behavior of the system will be studied by inspecting the power, voltage, frequency and power-delta curve. In addition to contingency situations, the effect of storage distribution on the different zones was studied.

Table 2. A list of the studied events

\begin{tabular}{lll}
\hline \hline No. & Event Description & Type \\
\hline 1 & Cable 1-2 outage & \\
2 & Cable 1-4 outage & Contingencies \\
3 & MTG2 1-2 outage & \\
4 & ATG2 1-2 outage & \\
5 & PMSM outage & Storage distribution \\
6 & Pulse load and storage at Zone 1 & \\
7 & Pulse load at Zone 1 and storage at Zone 4 & \\
8 & Pulse load at Zone 1 and storage distributed between Zones 1\&4 & \\
9 & Pulse load at Zone 1 and storage distributed between Zones 1, 2,3 \& 4 & \\
10 & Pulse load at Zone 1 without storage & \\
\hline \hline
\end{tabular}

\subsection{System security against contingency conditions}

\section{- Cable 1-2 outage}

In this case, buses 2, 3 and 4 as well as zones 2 and 4 encounter under voltage. The super capacitor tends to supply the demanded pulsed power once the pulses start due to its high power density. However, the power shared from the super capacitor drops before the end of the pulse due to its low energy density. The battery helps the super capacitor supply the pulsed loads. The system frequency and generators loading oscillations are within the limits, and there is no overloading reported. There is an under-voltage violation during transient, which infers that the system is not secure during this contingency event.

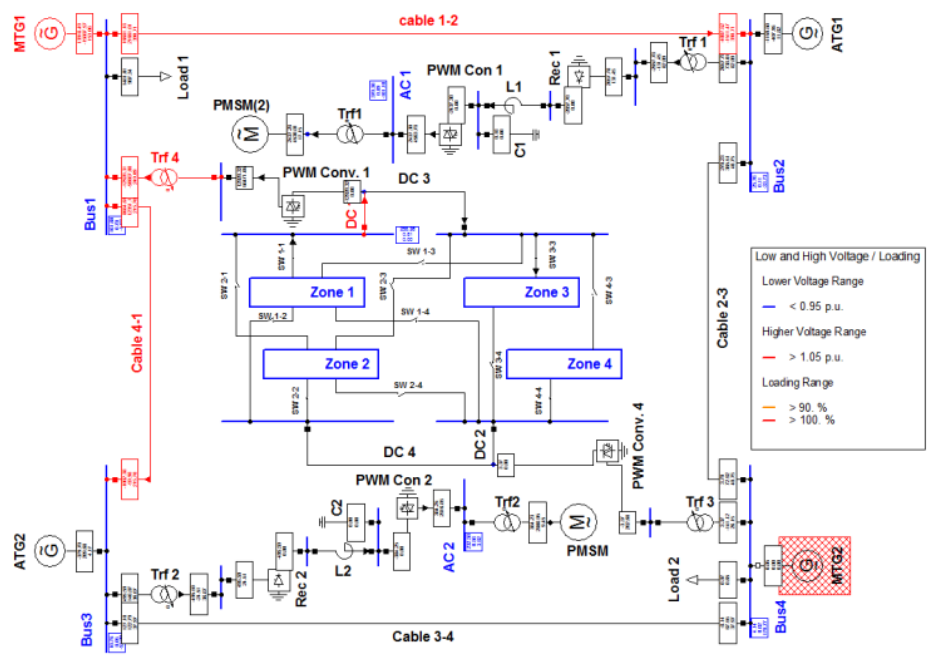

Fig. 7. System corresponding to MTG2 outage. 


\section{- Cable 1-4 outage}

Similar to the previous cases, the super capacitor is the fastest source to supply the pulses. Batteries contribute in all the four pulses to mitigate their effect on the generators. Under voltage violation happens during transients only at AC buses 3 and 4. No voltage violation occurred on the DC bus. Frequency oscillations are within the $\pm 0.5 \mathrm{~Hz}$ limit and there is no overloading reported.

\section{- Main turbine generator (MTG2) outage}

Figs. 7-10 present the results for a more severe case, which is the outage of one of the main units in this system (MTG2). There are several overvoltage and undervoltage conditioned reported. Moreover, the SC power oscillates significantly during the instability due to the outage of MTG2. Note that red lines represent overvoltage, whereas blue ones represent undervoltage. Frequency oscillations are out of the $\pm 0.5 \mathrm{~Hz}$ limit. Overloading is reported before the instability occurrence; hence it is very essential to monitor the loading of the generators dynamically to ensure the system security. It can be concluded that this contingency does not lead to system stable operation.

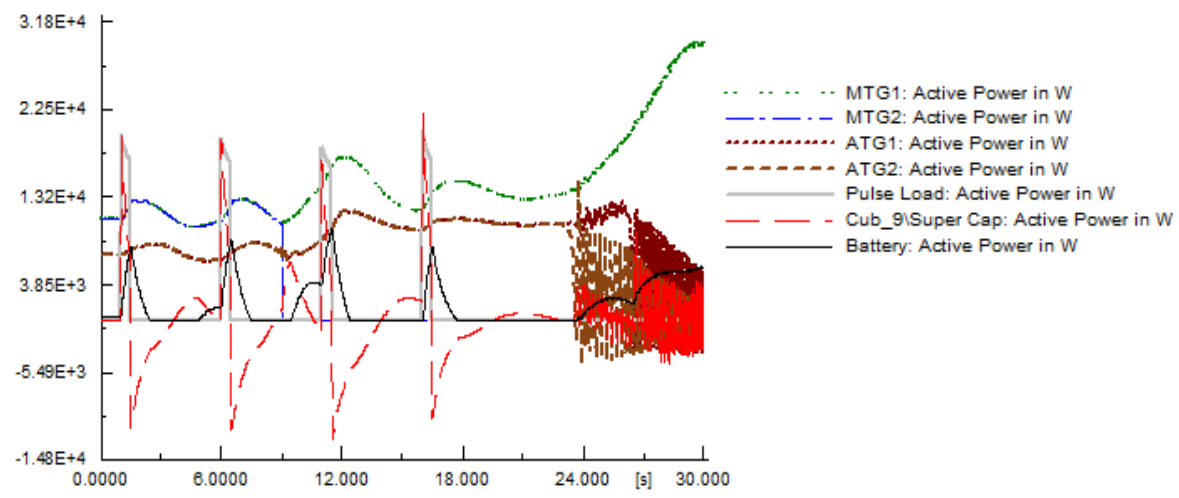

Fig. 8. Power sharing corresponding to MTG2 outage.
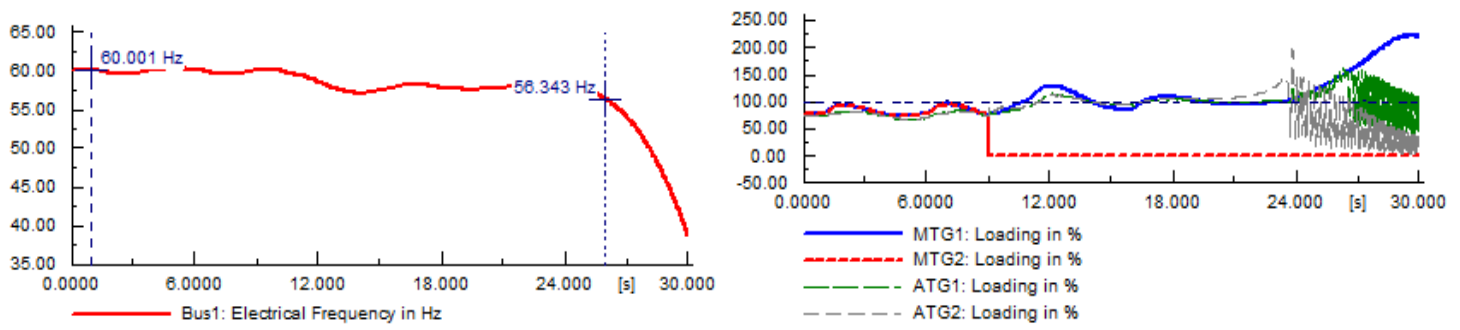

Fig. 9. Frequency and loading in case of MTG2 outage.
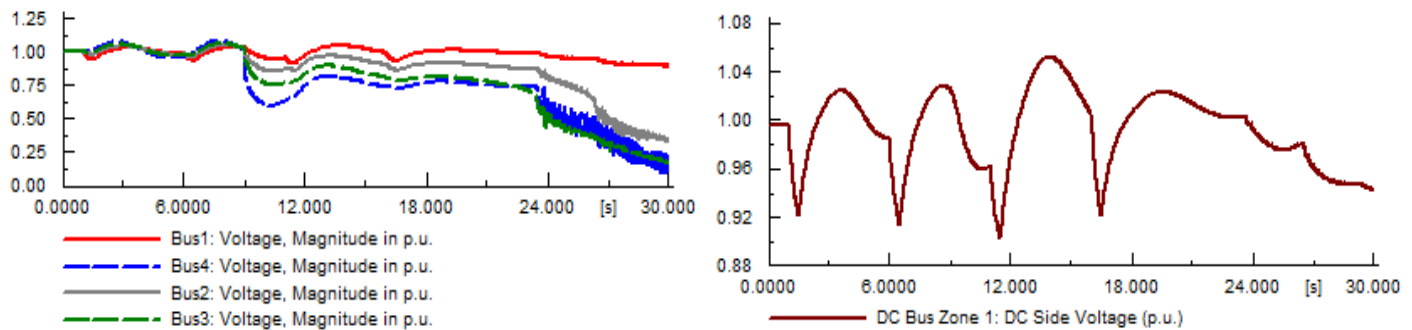

Fig. 10. Bus voltage due to MTG2 outage.

Therefore, the role of using energy storage systems on the case of MTG2 outage was studied by distributing energy storage at the four zones. The energy stored in the batteries was utilized to delay the instability, which gives more time for a remedial action. In this case the battery storage is distributed among all the four zones, and the oscillations of all the sources are within limits according to Figs. 11, 12. No voltage violation or loading problem was reported and the frequency is almost at its limits. 

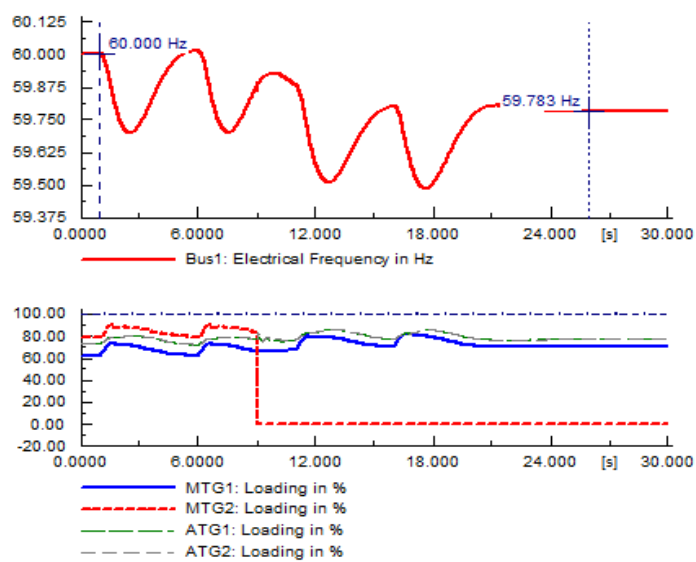

Fig. 11. Frequency and loading, battery distribution.

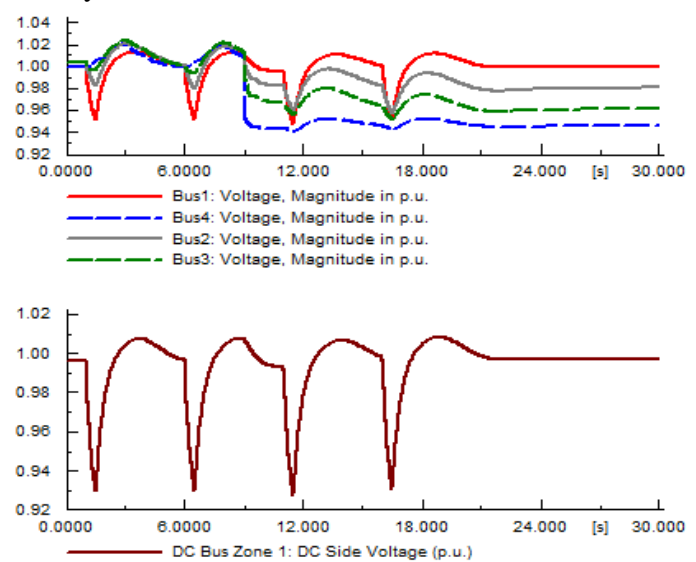

Fig. 12. Voltage fluctuations, battery distribution.

\section{- Auxiliary turbine generator (ATG2) outage}

The outage of ATG2 was also studied. Results show that the steady state values of the power shared from MTG1, ATG1 and ATG2 are higher than their initial values because of losing ATG2. Under voltage violation was reported during transient only at buses 3 and 4 . However, under frequency violation and overloading of MTG1, ATG1 and ATG2 are the main problems in this case. The rotor angle curves for the main generators show the system instability for this case.

\section{- Permanent magnet synchronous machine (PMSM) outage}

The last contingency study performed was related to the outage of one of the propulsion motors (PMSM). This case represents a challenging case on a shipboard power system since the loss of a big load is as problematic as the loss of generation in such a confined system. The loss of the load takes place in a very short time as compared to the time the mechanical system, controlling the prime mover of the generators, take to reach the new equilibrium point. The excess mechanical energy will lead the generator shaft to accelerate then fluctuate around the synchronous speed causing unwanted oscillations on the shipboard. In addition, it leads to problems on the power system in terms of frequency and loading. The DC bus voltage has no voltage violation. This reflects the impact of having the battery and super capacitor storage system on the DC bus. The steady state values of the power shared from all the generators are less than their initial values. Moreover, there was over frequency violation and overloading at the main turbine generator (MTG2), where the PMSM was connected. This overloading condition of MTG2 takes place for a very short time due to the oscillations in its rotor angle frequency. The rotor angle depicts no stability issue. However, the oscillations that take place after the disconnection of the permanent magnet synchronous propeller can be seen. 


\subsection{Effect of storage distribution on system dynamic security}

In this section, we will study the effect of distributing storages on the different zones of the system while the pulse load is occurring at the first zone. Distributing the storage has a significant impact on the reliability of the storage system. The aim of this study is to analyze the performance of distributed voltage control in different zones of microgrids. Hence, the pulsed load and super capacitor are connected to bus 1 and we distributed the storages over different zones based on the event studied.

\section{- Pulse load at Zone 1, storage bulked at Zone 1}

In this case, both the super capacitor and battery contribute to supplying the pulse load. No voltage violation was reported on AC or DC buses. The frequency of all the buses is within limits and no overloading was reported.

\section{- Pulse load at Zone 1, storage bulked at Zone 4}

The SC power oscillates more significantly than the previous case, and it takes more time to be charged (batteries are on another zone). Batteries start discharging not in synchronism with the pulse load. In addition, they do not supply the same amount of energy they can supply while connected at the same bus where the pulse load occurs. Overvoltage violation at busses 3 and 4 and under voltage violation at bus 1 and the DC bus are reported.

\section{- Pulse load at Zone 1, storage distributed over Zones 1 and 4}

In this case study, storage is distributed over two zones: zone 1 and zone 4 . Oscillations take place at bus 4 , similar to the previous case, the battery at the far bus (bus 4) starts discharging not in synchronism with the pulse load. While, the battery (or the portion of energy storage) at bus 1 significantly assists the super capacitor in mitigating the effect of the pulse load. No voltage, frequency or loading violation was reported in this case.

\section{- Pulse load at Zone 1, storage distributed over Zones 1, 2, 3 and 4}

The most reliable distribution of battery storage during sever conditions is when the storage is divided into all the four zones. All the generators encounter significantly less oscillations when the battery storage is distributed over the different zones. The batteries contribute to mitigating the pulse loads, the voltage oscillations are within the $\pm 10 \%$ limit and no frequency violation was reported and the loading of all the generators is within limits, and is relatively low.

Table 3 summarizes the various cases studied in this paper.

Table 3. Dynamic security analysis summary

\begin{tabular}{|c|c|c|c|c|c|c|}
\hline \# & Event Description & Voltage & Frequency & Loading & Security & $\begin{array}{c}\text { Event } \\
\text { Type }\end{array}$ \\
\hline 1 & Cable 1-2 Outage & 0 & $\bullet$ & $\bullet$ & Unsecure & \multirow{6}{*}{ 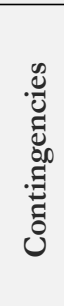 } \\
\hline 2 & Cable 1-4 Outage & $\mathbf{0}$ & $\bullet$ & $\bullet$ & Unsecure & \\
\hline 3 & MTG2 Outage & 0 & 0 & 0 & Unsecure & \\
\hline 4 & $\begin{array}{l}\text { MTG2 Outage with Energy } \\
\text { Storage Distribution }\end{array}$ & & 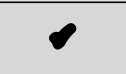 & $\curvearrowright$ & Secure & \\
\hline 5 & ATG2 Outage & $\mathbf{0}$ & $\mathbf{0}$ & $\mathbf{0}$ & Unsecure & \\
\hline 6 & PMSM Outage & $\boldsymbol{0}$ & $\boldsymbol{0}$ & (on limit) & Unsecure & \\
\hline 7 & Pulse Load and Storage at Zone 1 & $\bullet$ & $\bullet$ & $\bullet$ & Secure & \multirow{5}{*}{ 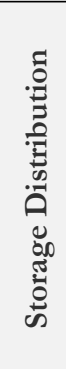 } \\
\hline 8 & $\begin{array}{l}\text { Pulse Load at Zone } 1 \text { and Storage } \\
\text { at Zone } 4\end{array}$ & 0 & $\boldsymbol{0}$ & $\boldsymbol{0}$ & Unsecure & \\
\hline 9 & $\begin{array}{l}\text { Pulse Load at Zone } 1 \text { and Storage } \\
\text { distributed between Zones } 1 \& 4\end{array}$ & 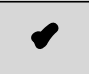 & $\bullet$ & $\bullet$ & Secure & \\
\hline 10 & $\begin{array}{l}\text { Pulse Load at Zone } 1 \text { and Storage } \\
\text { distributed between Zones } 1,2,3 \\
\& 4\end{array}$ & $\bullet$ & $\bullet$ & $\bullet$ & Secure & \\
\hline 11 & $\begin{array}{l}\text { Pulse Load at Zone } 1 \text { without } \\
\text { storage }\end{array}$ & 0 & - & - & Unsecure & \\
\hline
\end{tabular}




\section{Conclusion}

The security of islanded microgrids versus pulsed loads needs to be thoroughly investigated to assure stable operation, during normal as well as contingency operating conditions of islanded microgrids. Pulsed loads lead to power oscillations on the network, which can be mitigated if the microgrid is properly designed, and fast storage elements, such as super capacitors are utilized. However, when pulsed loads are accompanied with some contingencies, such as the loss of a main generator or load, the system may go out of step. Distributing the battery storage physically over multiple zones not only increases the reliability of the storage system during natural disasters, but also increases the security of the microgrid during pulsed loads and contingencies.

\section{References}

[1] Mohamed A, Mohammed O. Real-time energy management scheme for hybrid renewable energy systems in smart grid applications. Electric Power System Research, 2013; 96(1):133-143.

[2] Crider J, Sudhoff S. Reducing impact of pulsed power loads on microgrid power systems. IEEE Transactions on Smart Grid, 2010; 1(3):270-277.

[3] Kulkarni S, Santoso S. Impact of pulse loads on electric ship power system: With and without flywheel energy storage systems. IEEE Electric Ship Technologies Symposium, 2009; 1:568-573.

[4] Burgt J, Gelder P, Dijk E. Pulsed power requirements for future naval ships. In: Proc. of the 12th IEEE International Pulsed Power Conference, 1999:1357-1360.

[5] Mohamed A, Salehi V, Mohammed O. Real-time energy management algorithm for mitigation of pulse loads in hybrid microgrids. IEEE Transactions on Smart Grid, 2012; 3(4):1911-1922. 